\title{
Cognitive behavioural self help reduced binge eating in women
}

\author{
Carter JC, Fairburn CG. Cognitive-behavioral self-help for binge eating disorder: a controlled effectiveness study.J Consult Clin \\ Psychol 1998 Aug;66:616-23.
}

\section{Questions}

Is cognitive behavioural self help effective in women with binge eating disorder? Does pure self help (PSH) or guided self help (GSH) lead to a better outcome?

\section{Design}

Randomised controlled trial with 6 months follow up.

\section{Setting}

Community based study at Oxford University, UK.

\section{Participants}

72 women (mean age 40 y) who had at least weekly bulimic episodes (defined by the Eating Disorder Examination [EDE]) but had not used vomiting, fasting, laxatives, or diuretics to control their shape or weight during the previous 3 months. Exclusion criteria were $D S M-I V$ diagnosis of bulimia nervosa or anorexia nervosa, age $<18$ years or $>65$ years, pregnancy, medical disorder or treatment that influenced eating habits or weight, current psychiatric treatment, or previous treatment for binge eating. Follow up was $94 \%$ at 3 months and $86 \%$ at 6 months.

\section{Intervention}

Women were allocated to PSH, GSH, or a waiting list for 12 weeks, after which those on the waiting list $(\mathrm{n}=24)$ were allocated to PSH or GSH. Women in the PSH group $(n=35)$ followed a self help programme in a book.* Women in the GSH group $(n=34)$ attended 6-8 sessions of 25 minutes each in which a facilitator supported them in using the same self help book.

\section{Main outcome measure}

Frequency of binge eating was measured using the EDE.

\section{Main results}

After treatment, women in each self help group had fewer eating binges than those in the waiting list group (mean $4.3 v 13.5$ binge eating episodes $/ 28 \mathrm{~d}, \mathrm{p}=0.001$ for GSH and $9.3 v 13.5$ binge eating episodes/28 $\mathrm{d}, \mathrm{p}<0.05$ for PSH); the PSH and GSH groups did not differ in the frequency of binge eating. Cessation rates after treatment were greater in each self help group than in the waiting list group ( $p=0.001$ for GSH and $p=0.008$ for PSH) (table). Women in the GSH group had fewer eating binges than those in the PSH group at 3 and 6 months $(\mathrm{p}<0.05)$. No differences in the cessation rates existed between the 2 self help groups at 3 and 6 months (table).

\section{Conclusions}

Cognitive behavioural self help was effective in women with binge eating disorder. Guided self help led to a greater reduction in binge eating than did pure self help.

*Fairburn CG. Overcoming binge eating. New York: Guildford, 1995.

Binge eating cessation rates for guided self help (GSH), pure self help (PSH), and control (waiting list) $\dagger$

\begin{tabular}{llllll}
\hline Follow up & GSH & PSH & Control & RBI (95\% CI) & NNT (CI) \\
\hline After treatment & $50 \%$ & & $8 \%$ & $500 \%(82$ to 2129$)$ & $3(2$ to 6$)$ \\
After treatment & & $43 \%$ & $8 \%$ & $414 \%(53$ to 1828$)$ & $3(2$ to 9$)$ \\
After treatment & $50 \%$ & $43 \%$ & & $17 \%(-30$ to 96$)$ & Not significant \\
3 months & $41 \%$ & $37 \%$ & & $11 \%(-38$ to 100$)$ & Not significant \\
6 months & $50 \%$ & $40 \%$ & & $25 \%(-26$ to 114) & Not significant \\
\hline
\end{tabular}

†Abbreviations defined in glossary; RBI, NNT, and CI calculated from data in article.

Sources of funding: Wellcome Prize Studentship 38539 and Wellcome Principal Fellowship 46386. For correspondence: Prof C G Fairburn, Department of Psychiatry, University of Oxford, Warneford Hospital, Oxford OX3 $7 J X, U K$.

Abstract also published in Evidence-Based Nursing.

\section{Commentary}

Carter and Fairburn evaluated the relative effectiveness and utility of cognitive behavioral PSH and GSH in the treatment of binge eating disorder. The study mirrored conditions that would apply if these programmes were implemented in primary care or community settings. Results are encouraging, with both self help conditions leading to substantial reductions in binge eating. There is reason for confidence that GSH played a major part in symptom reduction; this conclusion, however, is more dubious for PSH. An impressive $68 \%$ of GSH participants reported following the entire self help programme compared with only $6 \%$ of the PSH group. Not surprisingly, PSH participants failed to show substantial reductions in dietary restraint-a theoretical cornerstone to overcoming binge eating according to the self help manual. Internal validity of the study is further threatened by participants who sought additional treatments. The PSH group was particularly prone to wanderlust, with 18\% seeking help for weight loss during treatment; during follow up, 43\% more sought weight loss help, 7\% sought additional help for eating problems, and 28\% sought help for other emotional problems (corresponding percentages for GSH were $0 \%$, $18 \%, 5 \%$, and $14 \%$, respectively). Fully $89 \%$ of the PSH group sought at least 1 additional treatment during self help treatment or follow up compared with $32 \%$ of GSH participants.

In summary, this study provides evidence that GSH may be a viable first step for individuals with binge eating disorder. Data are less convincing on the benefits ofsimply reading a self help book with no guidance. This study adds to the growing number that show that many patients with binge eating disorder and bulimia nervosa can be helped by minimal interventions. However, it appears to have tested the lower limit of treatment reductionism. The tendency for PSH participants to seek additional treatments may indicate that the relationship with a therapist or facilitator is critical to the change process. At this time, little evidence exists that the effectiveness of GSH can be generalised to anorexia nervosa which involves a more complicated treatment.

$$
\begin{array}{r}
\text { David M Garner, } \mathrm{PhD} \\
\text { Bowling Green State University and } \\
\text { University of Toledo } \\
\text { Toledo, Ohio, USA }
\end{array}
$$

